\title{
COVERING THE EDGES OF A GRAPH BY FOUR ODD SUBGRAPHS
}

\author{
MIRKO PETRUŠEVSKI
}

\begin{abstract}
A graph is odd if all its vertices have odd degrees. A Shannon triangle is a loopless graph on three pairwise adjacent vertices. If the parities of the sizes of its bouquets (of parallel edges) are denoted by $p, q, r$ in nonincreasing order, with 2 (resp. 1) denoting an even-sized (resp. odd-sized) bouquet, we then say the Shannon triangle is of type $(p, q, r)$. The minimum number of odd subgraphs which cover its edges is $p+q+r$. For a Shannon triangle of type $(2,2,2)$ (resp. $(2,2,1))$ this number equals 6 (resp. 5 ). We prove that, by excluding these two types of Shannon triangles, every other loopless connected graph admits an edge cover by four odd subgraphs.
\end{abstract}

\section{INTRODUCTION}

1.1. Terminology and notation. Throughout the article we mainly follow the terminology and notation used in $[1,9]$. A graph $G=(V(G), E(G))$ is always regarded as being finite (i.e. having finite sets of vertices $V(G)$, and of edges $E(G)$ ) with loops and parallel edges allowed. The maximum number of pairwise parallel edges is called multiplicity of $G$. Whenever $G$ is loopless and of multiplicity at most 1 , we say it is a simple graph. The parameters $n(G)=|V(G)|$ and $m(G)=|E(G)|$ are called order and size of $G$, respectively. For $X \subseteq V(G) \cup E(G)$, the subgraph of $G$ induced by $X$ is denoted with $G[X]$; on the other hand, the subgraph obtained by the removal of $X$ is denoted with $G-X$. We refer to each vertex $v$ of $G$ having an even (resp. odd) degree $d_{G}(v)$ as an even (resp. odd) vertex. In particular, if $d_{G}(v)$ equals 0 (resp. 1), we say that $v$ is an isolated (resp. pendant) vertex of $G$. A graph is called even (resp. odd) whenever all its vertices are even (resp. odd). An edge cover of $G$ is a family $\left\{H_{1}, \ldots, H_{k}\right\}$ of subgraphs of $G$ such that $\bigcup_{i=1}^{k} E\left(H_{i}\right)=E(G)$. Moreover, if the sets $E\left(H_{1}\right), \ldots, E\left(H_{k}\right)$ are pairwise disjoint, then $\left\{H_{1}, \ldots, H_{k}\right\}$ is a decomposition of $G$. An arbitrary mapping $\varphi: E(G) \rightarrow S$ is called edge-coloring of $G$, and then $S$ is the color set of $\varphi$. Whenever $|S|=k$, we say that $\varphi$ is a $k$-edge-coloring of $G$. For each color $c \in S, E_{c}(G, \varphi)$ denotes the color class of $c$, being the set of edges colored by $c$. In other words, $E_{c}(G, \varphi)=\varphi^{-1}(c)$, and whenever $G$ and $\varphi$ are clear from the context, we will use $E_{c}$ to denote the color class of $c$. Any decomposition $\left\{H_{1}, \ldots, H_{k}\right\}$ of $G$ can be interpreted as a

2010 Mathematics Subject Classification. 05C15.

Key words and phrases. Edge cover, odd subgraph, odd edge-coloring, Shannon triangle. 
$k$-edge-coloring of $G$ for which the color classes are $E\left(H_{1}\right), \ldots, E\left(H_{k}\right)$. For every $v \in V(G), E_{G}(v)$ denotes the set of edges incident to $v$. Given an edge-coloring of $G$ and a vertex $v$, we say the color $c$ appears at $v$ if $E_{c} \cap E_{G}(v) \neq \emptyset$.

In a connected graph $G$, a vertex $v$ is said to be a cut vertex if $G-v$ is disconnected. A block is a connected graph without cut vertices. Given a graph $G$, any maximal subgraph without cut vertices is called a block of $G$. So, if $B$ is a block of $G$, then $B$ as a graph has no cut vertex but $V(B)$ may contain cut vertices of $G$ (if $G$ is not a block, then $V(B)$ contains at least one cut vertex of $G$ ). For a block $B$ of $G$, if $V(B)$ contains at most one cut vertex of $G$, we say that $B$ is an end-block of $G$. Every connected graph $G$ which is not a block has at least two end-blocks. On the other hand, if $G$ is a block, then its only end-block is $G$ itself. Given a block $B$ of $G$, each $v \in V(B)$ which is not a cut vertex of $G$ is called an internal vertex of $B$. Note that any vertex $v$ of a block $G$, has a neighbor among the internal vertices of every end-block of $G-v$.

Given a connected graph $G$, an edge $e$ is said to be a bridge if $G-e$ is disconnected. Observe that whenever $e=v w$ is a bridge of $G$ and $w$ is not the only neighbor of $v$ in $G$, then $v$ is a cut vertex of $G$.

1.2. Edge covers and decompositions. Let $\mathcal{H}$ be a class of graphs and $G$ a given graph. The minimum size of an edge cover (resp. decomposition) of $G$ by members of $\mathcal{H}$ is denoted with $\operatorname{cov}_{\mathcal{H}}(G)$ (resp. $\operatorname{dec}_{\mathcal{H}}(G)$ ). Obviously, a decomposition of $G$ by members of $\mathcal{H}$ can be interpreted as an edge-coloring of $G$ such that each color class induces a member of $\mathcal{H}$. Usually, the following questions are of interest.

Problem 1. Does $G$ admit an edge cover (resp. decomposition) by members of $\mathcal{H}$ ? If so, what is the value of $\operatorname{cov}_{\mathcal{H}}(G)$ (resp. $\left.\operatorname{dec}_{\mathcal{H}}(G)\right)$ ? Does there exist $\max _{G} \operatorname{cov}_{\mathcal{H}}(G)$ (resp. $\max _{G} \operatorname{dec}_{\mathcal{H}}(G)$ ), taken over all the graphs $G$ admitting such edge covers (resp. decompositions)? If this maximum exists, for which graphs $G$ is it attained?

Historically speaking, the topic of Problem 1 started with the classical paper [2] on covering the edges of simple graphs by complete graphs. For the class $\mathcal{H}$ of even graphs, Problem 1 was answered by Matthews in [6]. The parity counter part is the case when $\mathcal{H}$ represents the class of odd graphs, and we will use $\mathcal{O}$ to denote it. The following result is proven in [7].

Theorem 1 (Pyber, 1991). For every simple graph $G$, it holds that $\operatorname{dec}_{\mathcal{O}}(G) \leq 4$.

It is easily checked that $W_{4}$, the wheel with 4 spokes, cannot be decomposed into less than 4 odd subgraphs. Hence, $\max _{G} \operatorname{dec}_{\mathcal{O}}(G)=4$, restricting to simple graphs $G$. This example can be used to construct an infinite series of related examples, as shown in [5]. The same paper contains the following result.

Theorem 2 (Matrai, 2006). For every simple graph $G$, it holds that $\operatorname{cov}_{\mathcal{O}}(G) \leq 3$.

As a straightforward consequence of the handshake lemma, no non-empty connected even graph of odd order can be covered by less than three odd subgraphs. 
Hence, the previous theorem implies that $\max _{G} \operatorname{cov}_{\mathcal{O}}(G)=3$, taken over the simple graphs $G$.

Neither of the last two results applies to the class of all graphs $G$. For example, Fig. 1 depicts four graphs (of order 3) with the following property: each of their odd subgraphs is of order 2 and size 1, i.e. a copy of $K_{2}$.
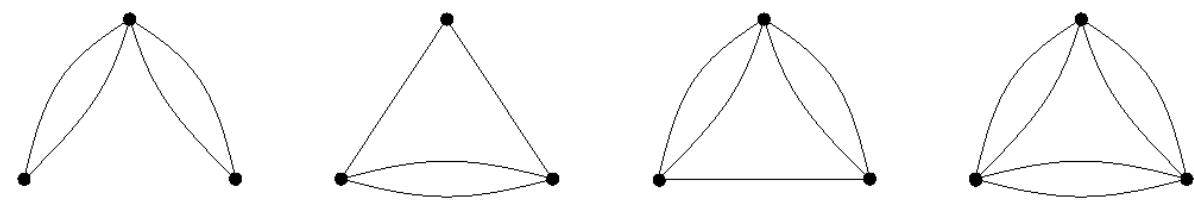

Figure 1. Four graphs $G$ having $\operatorname{dec}_{\mathcal{O}}(G)=\operatorname{cov}_{\mathcal{O}}(G)$ equal to $4,4,5$, and 6 , respectively.

Presented in the context of edge-colorings, $\operatorname{dec}_{\mathcal{O}}(G)$ naturally gives rise to the following notion. An odd edge-coloring of a graph $G$ is a (not necessarily proper) edge-coloring such that each color class induces an odd subgraph of $G$. If it uses at most $k$ colors, we say it is an odd $k$-edge-coloring, and then $G$ is odd $k$-edgecolorable. Whenever $G$ admits odd edge-colorings, the odd chromatic index $\chi_{o}^{\prime}(G)$ is defined as the minimum integer $k$ for which $G$ is odd $k$-edge-colorable. In other words, $\chi_{o}^{\prime}(G)=\operatorname{dec}_{\mathcal{O}}(G)$.

By definition, at a vertex $v$ each loop colored by $c$ contributes 2 to the count of appearances of the color $c$ at $v$ (i.e. on the edges belonging to $E_{G}(v)$ ). Thus, it is obvious that a necessary and sufficient condition for the existence of an odd edge-coloring of $G$ is the absence of vertices incident only to loops. Clearly, the same condition is necessary and sufficient for the existence of an edge cover of $G$ by odd subgraphs. Apart from this, the presence of loops does not influence the existence nor changes the values of the indices $\chi_{o}^{\prime}(G)$ and $\operatorname{cov}_{\mathcal{O}}(G)$. Therefore, while studying these matters, it is no loss of generality to restrict to connected loopless graphs. The rightmost graph in Fig.1 shows the existence of graphs $G$ with $\chi_{o}^{\prime}(G)=\operatorname{cov}_{\mathcal{O}}(G)=6$.

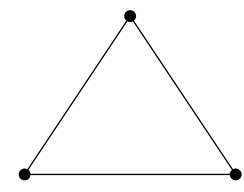

$(1,1,1)$

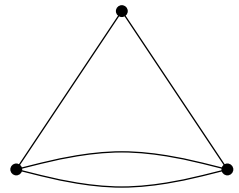

$(2,1,1)$

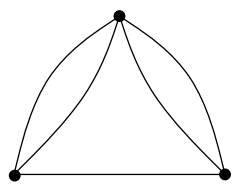

$(2,2,1)$

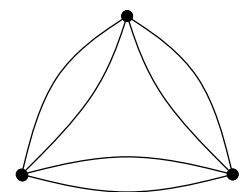

$(2,2,2)$

Figure 2. Four Shannon triangles (the smallest one of each type).

For a loopless graph $G$, a bouquet is the complete set of edges between a pair of adjacent vertices. As defined in [4], a Shannon triangle is a loopless graph on 
three pairwise adjacent vertices. Observe that for any Shannon triangle, the edge set of every odd subgraph is fully contained in a single bouquet. Let $p, q, r$ be the parities of the sizes of its bouquets in non-increasing order, with 2 (resp. 1) denoting an even-sized (resp. odd-sized) bouquet. Then $G$ is a Shannon triangle of type $(p, q, r)$. Fig. 2 depicts the smallest Shannon triangle of each type. In regard to the odd chromatic index of loopless graphs, the following result is proven in [4].

Theorem 3. For every connected loopless graph $G$, it holds that $\chi_{o}^{\prime}(G) \leq 6$. Moreover, equality holds if and only if $G$ is a Shannon triangle of type $(2,2,2)$.
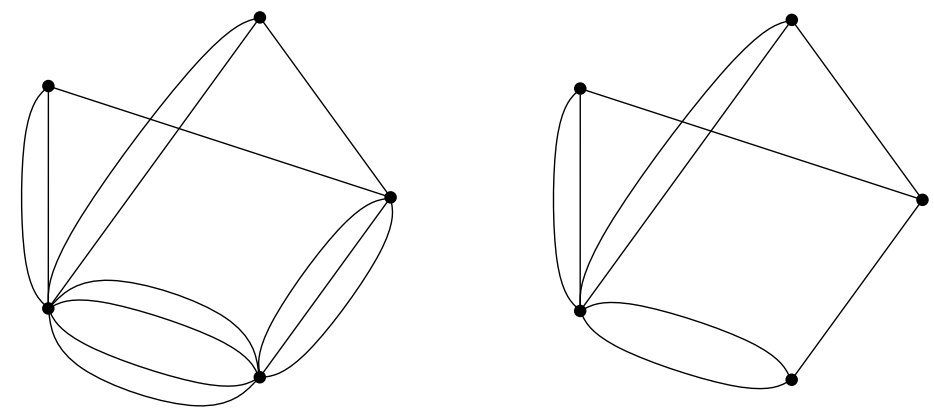

Figure 3. A loopless graph (left) and its reduction (right).

In [4], the reduction $\operatorname{red}(G)$ of a loopless graph $G$ is defined to be a spanning subgraph obtained by the following intervention at every bouquet: remove maximum possible even number of edges from the bouquet without altering the adjacency relation in $V(G)$ (see Fig. 3 for an example). Obviously, $\operatorname{red}(G)$ is of multiplicity at most 2, and up to isomorphism, each loopless graph $G$ has a unique reduction. Observe that if $G$ is a Shannon triangle, then $\operatorname{red}(G)$ is of the same type as $G$. We say that a loopless graph $G$ is reduced whenever its multiplicity is at most 2, i.e. when $G \cong \operatorname{red}(G)$. The former of the inequalities $(*)$ was shown in [4], and the latter can be proved in a similar fashion.

$$
\chi_{o}^{\prime}(G) \leq \chi_{o}^{\prime}(\operatorname{red}(G)) \quad \text { and } \quad \operatorname{cov}_{\mathcal{O}}(G) \leq \operatorname{cov}_{\mathcal{O}}(\operatorname{red}(G)) .
$$

Having in mind how the odd subgraphs of every Shannon triangle look like, the following result is straightforward.

Proposition 4. Given a Shannon triangle $G$ of type $(p, q, r)$, it holds that

$$
\chi_{o}^{\prime}(G)=\operatorname{cov}_{\mathcal{O}}(G)=p+q+r .
$$

Since the inequality $\operatorname{cov}_{\mathcal{O}}(G) \leq \chi_{o}^{\prime}(G)$ clearly holds in general, the last proposition and Theorem 3 provide an answer to Problem 1 when $\mathcal{H}=\mathcal{O}$. The next conjecture was proposed in [4].

Conjecture 1. Let $G$ be a connected loopless graph which is not a Shannon triangle of type $(2,2,2)$ or $(2,2,1)$. Then $\chi_{o}^{\prime}(G) \leq 4$. 
Supporting Conjecture 1, in this paper we prove the following result.

Theorem 5. Let $G$ be a connected loopless graph which is not a Shannon triangle of type $(2,2,2)$ or $(2,2,1)$. Then $\operatorname{cov}_{\mathcal{O}}(G) \leq 4$.

To supply a proof of Theorem 5 , we will use methods such as eliminating odd forests or co-forests, conditions on connectivity etc., developed in $[5,3]$. The rest of the article is divided into three sections. In the next one several lemmas are collected. At the beginning of Section 3, the existence of edge covers by odd subgraphs is studied for some particular graphs. Hopefully this approach makes the proof of Theorem 5, presented at the end of that section, more transparent. In the final section, two related conjectures are stated and briefly discussed.

\section{Preliminary Results}

We begin by recalling the definition of a $T$-join. For a graph $G$, let $T$ be an even-sized subset of $V(G)$. Following [1], a spanning subgraph $H$ of $G$ is said to be a $T$-join if $d_{H}(v)$ is odd for all $v \in T$ and even for all $v \in V(G) \backslash T$. For example, if $P$ is an $x y$-path in $G$, the spanning subgraph of $G$ with edge set $E(P)$ is an $\{x, y\}$-join. Observe that by taking the symmetric difference $H \oplus K$ of a $T$-join $H$ and a spanning even subgraph $K$, we again obtain a $T$-join. In particular, removal (resp. addition) of the edges of an edge-disjoint cycle from (resp. to) a $T$-join, furnishes a $T$-join. Thus, whenever a $T$-join of $G$ exists, there also exists such a forest (resp. co-forest). By the handshake lemma, a necessary condition for the existence of a $T$-join is that $T$ intersects every component of $G$ in an even number of vertices (possibly equal to zero). The following classical result about $T$-joins claims that this condition also suffices (see [8]).

Proposition 6. Given a connected graph $G$ and an even-sized subset $T$ of $V(G)$, there exists a $T$-join of $G$.

Corollary 7. Given a connected graph $G$ and an even-sized subset $T$ of $V(G)$, there exists a $T$-join $H$ of $G$ that is a co-forest.

Corollary 8. Given a connected graph $G$ of even order, there exists an odd coforest in $G$.

Whenever the graph $G$ is clear from the context, the edge-complement of a subgraph $H$ is denoted by $\hat{H}$, i.e. $\hat{H}=G-E(H)$. Now we state a lemma that appears in [5]. For the sake of completeness, we provide another proof of it here.

Lemma 9. Given are a forest $F$, a vertex $w \in V(F)$, and a parity $\pi$ which is even if $w$ is isolated. Then, $F$ admits an edge-coloring with the color set $\{1,2\}$ which satisfies the following two conditions:

(1) the subgraph $F_{1}=F\left[E_{1}\right]$ is odd;

(2) for the edge-complement $F_{2}=\hat{F}_{1}$ it holds that $d_{F_{2}}(w)=\pi(\bmod 2)$ and $d_{F_{2}}(v)$ is odd or zero, for each $v \neq w$. 
Proof. We may assume $F$ is a tree. In case $d_{F}(w)=\pi(\bmod 2)$, construct a 2-edgecoloring of $F$ as follows: color $E_{F}(w)$ by 2 ; as long as $E(F)$ is not fully colored, select a non-pendant vertex $v$ with just one incident edge colored so far, and extend the current edge-coloring to $E_{F}(v)$ so that each color from $\{1,2\}$ appears odd or zero number of times at $v$. Since $F$ is connected and acyclic, by repeating this procedure we end up with a 2-edge-coloring of $F$ which meets the requirements of the lemma. Consider now the case when $d_{F}(w) \neq \pi(\bmod 2)$, hence $d_{F}(w)>0$. Select an $e \in E_{F}(w)$. Color $E_{F}(w) \backslash\{e\}$ by 2 and color the edge $e$ by 1 . Extend this edge-coloring of $E_{F}(w)$ to a 2-edge-coloring of $F$ as described in the previous case.

As an immediate consequence of Lemma 9, we have the following result.

Corollary 10. For every forest $F$, it holds that $\chi_{o}^{\prime}(F) \leq 2$.

Using this corollary, we can easily show that every connected graph of even order is odd 3-edge-colorable.

Corollary 11. For every connected graph $G$ of even order, it holds that $\chi_{o}^{\prime}(G) \leq 3$.

Proof. Let $H$ be a $V(G)$-join of $G$ which is also a co-forest. Take an odd edgecoloring of $\hat{H}$ with the color set $\{1,2\}$ and extend it to $E(G)$ by coloring $E(H)$ with 3 . We thus obtain an odd 3-edge-coloring of $G$.

Consider now a reduced graph $G$. For an arbitrary $v \in V(G)$, we denote by $N_{G}(v)$ the set of neighbors of $v$ in $G$. Distinguish between two types of neighbors $u \in N_{G}(v)$ : those for which there is a unique $u v$-edge in $G$, and those for which there are two $u v$-edges in $G$. We introduce notation $N_{G}^{\prime}(v)$ and $N_{G}^{\prime \prime}(v)$ to denote the respective subsets of $N_{G}(v)$. Often, we will use notation $v \sim u, v \approx u$, or $v \leftrightarrow u$, to depict the relationship $u \in N_{G}^{\prime}(v), u \in N_{G}^{\prime \prime}(v)$, or $u \in N_{G}(v)$, respectively. Clearly, $v$ is an odd vertex of $G$ if and only if $N_{G}^{\prime}(v)$ is odd-sized. The next lemma deals with a reduced graph $G$ all of whose cycles (if any) share a vertex $v$.

Lemma 12. Given a reduced graph $G$, let $v \in V(G)$ be such that $G-v$ is a forest. Then, $G$ admits an edge-coloring with the color set $\{1,2\}$ so that in both $G\left[E_{1}\right]$ and $G\left[E_{2}\right]$, each vertex distinct from $v$ is of odd degree.

Proof. Let $N_{G}^{\prime}(v)=\left\{u_{1}, \ldots, u_{s}\right\}$ and $N_{G}^{\prime \prime}(v)=\left\{w_{1}, \ldots, w_{t}\right\}$. Consider the graph $F$ obtained from $G-v$ by adding to each $u_{i}$ one pendant edge linking it to a new pendant vertex $u_{i}^{\prime}$, and adding to each $w_{j}$ two pendant edges linking it to two new pendant vertices $w_{j}^{\prime}, w_{j}^{\prime \prime}$. Clearly $F$ is a forest, and we may take an odd edge-coloring of $F$ with the color set $\{1,2\}$. Identify $u_{1}^{\prime}, \ldots, u_{s}^{\prime}, w_{1}^{\prime}, w_{1}^{\prime \prime}, \ldots, w_{t}^{\prime}, w_{t}^{\prime \prime}$ into a vertex $v$, while keeping the colors on the edges. We have thus regained $F$ with a required edge-coloring.

Regarding the previous lemma, if we additionally assume that $v$ is an odd vertex of $G$, we deduce the following corollary. 
Corollary 13. Given a reduced graph $G$, let $v$ be an odd vertex of $G$ such that $G-v$ is a forest. Then, $G$ admits an edge-coloring with the color set $\{1,2\}$ so that the subgraph $F_{1}=G\left[E_{1}\right]$ is odd, while in its edge-complement $F_{2}=\hat{F}_{1}, d_{F_{2}}(v)$ is even whereas for each vertex $u \neq v, d_{F_{2}}(u)$ is odd or zero.

To prove the next lemma, we will use a previously mentioned fact that the symmetric difference of a $T$-join and a spanning even subgraph is again a $T$-join of the considered graph.

Lemma 14. In a connected reduced graph $G$, let $v \in V(G)$ be a non-cut vertex, $u \in N_{G}(v)$, and $T \subseteq V(G)$ be even-sized. Then, $G$ has a $T$-join $C$ which is a co-forest, with the following two additional properties holding for $v$ in the edgecomplement $\hat{C}$ :

(i) If either $d_{G}(v)$ is odd and $v \in T$ or $d_{G}(v)$ is even and $v \notin T$, then $d_{\hat{C}}(v)=$ 0.

(ii) If either $d_{G}(v)$ is odd and $v \notin T$ or $d_{G}(v)$ is even and $v \in T$, then $d_{\hat{C}}(v)=$ 1 with $N_{\hat{C}}(v)=\{u\}$.

Proof. The requirement that $C$ is a co-forest can be easily achieved once the rest is fulfilled. Namely, suppose $C$ is a $T$-join satisfying the two additional properties for $v$ in the edge-complement $\hat{C}$. Then, in case $C$ is not a co-forest, we add to $E(C)$ the edges of a cycle appearing in $\hat{C}$. By doing this, both the property of $C$ being a $T$-join and the additional properties for $v$ in the edge-complement are preserved. Repetition of this procedure, eventually gives a co-forest which meets all the requirements.

(i) Since $T$ is even-sized, a $T$-join $H$ of $G$ exists. Observe that $v$ is an even vertex of the edge-complement $\hat{H}$. If $d_{\hat{H}}(v)=0$ we are done, hence assume $d_{\hat{H}}(v)>0$ and let $S=N_{\hat{H}}^{\prime}(v)$. Since $G-v$ is connected, it has an $S$-join. Therefore, there exists an $S$-join $K_{S}$ of $G$ such that $d_{K_{S}}(v)=0$. Take the symmetric difference of $K_{S}$ and the spanning subgraph of $G$ with edge set $E_{\hat{H}}(v)$. The obtained graph $K$ is an even spanning subgraph of $G$ for which $E_{K}(v)=E_{\hat{H}}(v)$. Thus, $C=H \oplus K$ is a $T$-join of $G$ such that $d_{\hat{C}}(v)=0$.

(ii) For an arbitrary $T$-join $H$ of $G, d_{\hat{H}}(v)$ is odd, i.e. $N_{\hat{H}}^{\prime}(v)$ is odd-sized. Let $e$ be a $u v$-edge in $G$ and let $S=N_{\hat{H}}^{\prime}(v) \triangle\{u\}$. Take an $S$-join $K_{S}$ of $G$ such that $d_{K_{S}}(v)=0$. Denote by $K$ the symmetric difference of $K_{S}$ and the spanning subgraph of $G$ with edge set $E_{\hat{H}}(v) \triangle\{e\}$. Observe that $K$ is an even spanning subgraph of $G$ for which $E_{K}(v)=E_{\hat{H}}(v) \triangle\{e\}$. Thus, $C=H \oplus K$ is a $T$-join of $G$ for which $d_{\hat{C}}(v)=1$ and $e \in E(\hat{C})$.

\section{Proof of Theorem 4}

We begin by considering several particular cases of the theorem. The next two lemmas study a connected graph $G$ having a specific end-block $B$.

Lemma 15. If a connected graph $G$ has an odd end-block $B$, then $\chi_{o}^{\prime}(G) \leq 3$. 
Proof. By Corollary 11, we may assume that $G$ is of odd order. Let $s$ be the only cut vertex of $G$ belonging to $V(B)$. Since $V(B)$ is even-sized (by the handshake lemma), the connected graph $G^{\prime}=G-(V(B) \backslash\{s\})$ is of even order. By Corollary 7, an odd co-forest $C$ exists in $G^{\prime}$. Take an edge-coloring of the edgecomplement $F=G^{\prime}-E(C)$ as in Lemma 9 with $s$ in the role of $w$ and $\pi$ even. Extend this edge-coloring to $E(G)$ by coloring $E(B)$ with 2 , and $E(C)$ with 3 . We have thus constructed an odd 3-edge-coloring of $G$.

Lemma 16. Let $G$ be a connected graph which is not a block, and has a Shannon triangle of type $(2,2,2),(2,2,1)$, or $(2,1,1)$ as an end-block $B$. Then $\operatorname{cov}_{\mathcal{O}}(G) \leq 4$.

Proof. By the second inequality of $\left(^{*}\right)$ and Corollary 11 , we may assume $G$ is a reduced graph of odd order. Hence, $B$ is one of the graphs depicted in Fig. 2 excepting the leftmost one. Let $s$ be the only cut vertex of $G$ belonging to $V(B)$. We consider first the case when $d_{B}(s) \geq 3$. Denote by $v, w$ the other two vertices of $B$ so that $v \approx s$, and let $e$ be an edge incident to $s$ in $G-\{v, w\}$. Denote by $f, g$ the $v s$-edges of $G$, and let $h$ be a $v w$-edge ( $h$ might be the unique $v w$ edge of $G$ or there might be another one, denoted by $\left.h^{*}\right)$. The connected graph $G^{\prime}=G-v$ is of even order, hence it admits an odd edge-coloring $\varphi^{\prime}$ with the color set $\{1,2,3\}$. Since $d_{G^{\prime}}(w) \leq 2$, we may permute (if necessary) the colors of $\varphi^{\prime}$ so that 3 does not appear at $w$. Let $H_{1}^{\prime}, H_{2}^{\prime}, H_{3}^{\prime}$ be the subgraphs induced by the color classes $E_{1}, E_{2}, E_{3}$, respectively. Define $H_{4}=G[\{e, f, g, h\}]$. If $v \sim w$, then set $H_{1}=H_{1}^{\prime}, H_{2}=H_{2}^{\prime}, H_{3}=H_{3}^{\prime}$. Otherwise, $v \approx w$ and then set $H_{1}=H_{1}^{\prime}$, $H_{2}=H_{2}^{\prime}, H_{3}=H_{3}^{\prime}+h^{*}$.

Consider now the case when $d_{B}(s)=2$. Then $B$ must be the second (from the left) graph of Fig. 2. Denote by $v, w$ the other two vertices of $B$, and let $f=s v$, $g=s w$, while $h$ and $h^{*}$ are the two $v w$-edges. Once again, let $e$ be an edge incident to $s$ in $G-\{v, w\}$. Consider an odd edge-coloring $\varphi^{\prime}$ of $G^{\prime}=G-v$ with the color set $\{1,2,3\}$. Since $d_{G^{\prime}}(w)=1$ we may permute (if necessary) the colors of $\varphi^{\prime}$ so that only the color 1 appears at $w$. Let $H_{1}^{\prime}, H_{2}^{\prime}, H_{3}^{\prime}$ be the subgraphs induced by the color classes $E_{1}, E_{2}, E_{3}$, respectively. Set $H_{1}=H_{1}^{\prime}, H_{2}=H_{2}^{\prime}+h$, $H_{3}=H_{3}^{\prime}+h^{*}$, and $H_{4}=G[\{e, f, g\}]$.

In both cases, $\left\{H_{1}, H_{2}, H_{3}, H_{4}\right\}$ is an edge cover of $G$ by odd subgraphs.

In the next lemma, we look at a reduced connected graph $G$ having a specific vertex $w$.

Lemma 17. Let $G$ be a reduced connected graph which is not a Shannon triangle of type $(2,2,2)$ or $(2,2,1)$. Let $v$ be a non-cut vertex of $G$ such that $\left|N_{G}(v)\right| \leq 2$ and $N_{G}^{\prime \prime}(v) \neq \emptyset$. Then $\operatorname{cov}_{\mathcal{O}}(G) \leq 4$.

Proof. We may assume $G$ is of odd order. Consider first the case when $\left|N_{G}(v)\right|=1$, and let $w$ be the only neighbor of $v$. Take an odd co-forest $C$ in $G-v$, and let $F=G-v-E(C)$ be its edge-complement. There exists a 2-edge-coloring $\varphi$ of $F$ as in Lemma 9 with $\pi$ even. Extend $\varphi$ to $E(G)$ by coloring one $v w$-edge with 2, $E(C)$ with 3 , and the other $v w$-edge with 4 . This furnishes an odd 4-edge-coloring of $G$. 
Next, we look at the case when $\left|N_{G}(v)\right|=2$. Let $w \approx v$ and $u$ be the other neighbor of $v$. If $N_{G}(w) \subseteq\{u, v\}$, then unless $G$ is a Shannon triangle of type $(2,1,1)$, the previous case or Lemma 16 applies. Therefore we may assume the existence of an edge $e \in E_{G}(w) \backslash\left(E_{G}(u) \cup E_{G}(v)\right)$. First, we consider $d_{G}(v)=3$. Denote by $f, g$ the $v w$-edges and let $h$ be the unique $u v$-edge. For an odd edgecoloring of $G-v$ with the color set $\{1,2,3\}$, let $H_{1}, H_{2}, H_{3}$ be the subgraphs induced by the respective color classes, and define $H_{4}=G[\{e, f, g, h\}]$. Second, we consider $d_{G}(v)=4$. Once again, let $f, g$ be the $v w$-edges and $h, k$ be the $u v$ edges. There exists a edge-coloring of $G-v$ with the color set $\{1,2,3\}$ such that the color classes $E_{1}$ and $E_{3}$ respectively induce odd subgraphs $H_{1}^{\prime}$ and $H_{3}^{\prime}$, whereas for the subgraph $H_{2}^{\prime}$ induced by $E_{2}$ either $u \notin V\left(H_{2}^{\prime}\right)$ and $H_{2}^{\prime}$ is odd, or $u$ is the only even vertex of $H_{2}^{\prime}$. Set $H_{1}=H_{1}^{\prime}, H_{2}=H_{2}^{\prime}+k, H_{3}=H_{3}^{\prime}, H_{4}=G[\{e, f, g, h\}]$.

On both occasions, $\left\{H_{1}, H_{2}, H_{3}, H_{4}\right\}$ is an edge cover of $G$ by odd subgraphs.

The following lemma assumes the existence of a specific pair $v, w$ of adjacent vertices in a connected graph $G$.

Lemma 18. Let $G$ be a connected graph which is not a Shannon triangle of type $(2,2,2)$ or $(2,2,1)$. Let $v, w \in V(G)$ be two adjacent vertices such that $v$ is an even non-cut vertex of $G$, and $w$ is a non-cut vertex of $G-v$.

(i) If $v \sim w$, then $\chi_{o}^{\prime}(G) \leq 4$. Moreover, if $d_{G}(w)$ is even then $\chi_{o}^{\prime}(G) \leq 3$.

(ii) If $v \approx w$, then $\operatorname{cov}_{\mathcal{O}}(G) \leq 4$.

Proof. By the second inequality of $\left(^{*}\right)$ and Corollary 11 , we may assume $G$ is a reduced graph of odd order.

(i) Denote by $e$ the unique $v w$-edge of $G$. Assume first that $d_{G}(w)$ is odd, hence $d_{G-v}(w)$ is even. By Lemma 14, $G-v$ contains an odd co-forest $C$ such that $d_{F}(w)=1$ in the edge-complement $F=G-v-E(C)$. Let $f$ be the only edge in $E_{F}(w)$. For the graph $G^{\prime}=G-(E(C) \cup\{e, f\})$ it holds that $G^{\prime}-v$ is a forest, $d_{G^{\prime}}(v)$ is odd, and $d_{G^{\prime}}(w)=0$. Take an edgecoloring of $G^{\prime}$ as in Corollary 13, and extend it to an odd 4-edge-coloring of $G$ by coloring $e$ with $2, E(C)$ with 3 , and $f$ with 4 .

Assume now that $d_{G}(w)$ is even. This time, $d_{G-v}(w)$ is odd, hence $G-v$ contains an odd co-forest $C$ such that $d_{F}(w)=0$ in the edge-complement $F=(G-v)-E(C)$. Consider the graph $G^{\prime}=G-(E(C) \cup\{e\})$ and proceed as above to obtain an odd 3-edge-coloring of $G$.

(ii) It is enough to look at the case when $d_{G}(v) \geq 4$, for otherwise Lemma 17 applies. Let $u$ be a neighbor of $v$ different from $w$. By Lemma 17, we may assume there exists an $s \in N_{G}(w) \backslash\{v, u\}$, and let $e$ be an $s w$-edge. Denote by $f, g$ the two $v w$-edges, and by $h$ any $u v$-edge. We consider separately the two possible parities of $d_{G}(w)$ :

- The degree $d_{G}(w)$ is odd. Note that $h$ is not a bridge of $G$. In the connected graph $G^{\prime}=G-\{f, h\}$ both $v$ and $w$ are even vertices, with $v \sim w$. By part $(i), G^{\prime}$ admits an odd 3-edge-coloring. Let 
$H_{1}, H_{2}, H_{3}$ be the subgraphs induced by the color classes, and define $H_{4}=G[\{e, f, g, h\}]$.

- The degree $d_{G}(w)$ is even. Let $G^{\prime}=G-\{e, f, h\}$. Since $d_{G-v}(w) \geq 2$ and $w$ is a non-cut vertex of $G-v$, the edge $e$ is not a bridge of $G-v$. Therefore, $G^{\prime}$ is connected, and $d_{G^{\prime}}(w)$ is even, hence part $(i)$ applies. Let $H_{1}, H_{2}, H_{3}$ be the subgraphs induced by the color classes of an odd 3-edge-coloring of $G^{\prime}$, and define $H_{4}=G[\{e, f, g, h\}]$.

On both occasions, $\left\{H_{1}, H_{2}, H_{3}, H_{4}\right\}$ is an edge cover of $G$ by odd subgraphs.

We are now ready for the proof of Theorem 5 .

Proof. By the second inequality of $(*)$ and Corollary 11, it suffices to consider reduced graphs of odd order. We prove the theorem by induction on $n(G)$. If $n(G)=3$, the statement follows from $m(G) \leq 4$. Let $n$ be an odd integer greater than 3. Assume the theorem holds for reduced graphs of order less than $n$, and let $G$ be a reduced connected graph of order $n$ which is not a Shannon triangle of type $(2,2,2)$ or $(2,2,1)$. We consider first the case when $G$ is a block. By the handshake lemma, there exists an even vertex $v$ of $G$. If $G-v$ is also a block, then for an arbitrary neighbor $w$ of $v$ Lemma 18 applies. Assume $G-v$ is not a block, and look at an arbitrary end-block $B$ of $G-v$. Denote by $s$ the only cut vertex of $G$ belonging to $V(B)$. As remarked at the end of Subsection 1.1, the set $V(B) \backslash\{s\}$ contains a neighbor $w$ of $v$, and therefore Lemma 18 applies.

Consider now the case when $G$ is not a block, and denote by $B$ an end-block of $G$. Let $s$ be the only cut vertex of $G$ belonging to $V(B)$. We distinguish two possibilities for the internal vertices of $B$.

(a) Every internal vertex of $B$ is odd. We may assume that $d_{B}(s)$ is even, for otherwise Lemma 15 implies the inequality $\chi_{o}^{\prime}(G) \leq 3$. Consider the graph $G^{\prime}=G-(V(B) \backslash\{s\})$. By Lemma 16, we may assume that $G^{\prime}$ is not a Shannon triangle of type $(2,2,2)$ or $(2,2,1)$. Hence, the induction hypothesis implies the existence of an edge cover $\left\{H_{1}^{\prime}, H_{2}^{\prime}, H_{3}^{\prime}, H_{4}^{\prime}\right\}$ of $G^{\prime}$ by odd subgraphs. Moreover, by permuting indices if necessary, we may assume that $d_{H_{1}^{\prime}}(s)>0$. Define $H_{1}=H_{1}^{\prime} \cup B, H_{2}=H_{2}^{\prime}, H_{3}=H_{3}^{\prime}$, $H_{4}=H_{4}^{\prime}$. These four odd subgraphs constitute an edge cover of $G$.

(b) At least one internal vertex $v$ of $B$ is even. Hence, $v$ is an even non-cut vertex of $G$. Assume first that $B-v$ is a block. Then $N_{G}(v) \neq\{s\}$, and $B-v$ is an end-block of $G-v$. Take a neighbor $w$ of $v$ among the internal vertices of $B-v$ and apply Lemma 18.

Assume now that $B-v$ is not a block. In every end-block of $B-v$, an internal vertex is adjacent to $v$. There exists an end-block $B^{\prime}$ of $B-v$ which does not contain $s$ as an internal vertex. Thus $B^{\prime}$ is an end-block of $G-v$ as well. Take an internal vertex $w$ of $B^{\prime}$ which is adjacent to $v$. Then $w$ is a non-cut vertex of $G-v$ and Lemma 18 once again applies.

This completes the proof of Theorem 5 . 


\section{Concluding Remarks And Further WOrK}

The first two of the four graphs depicted in Fig. 1 assure that the inequality of Theorem 5 is sharp. As already mentioned in the Introduction, one improvement of our main result would be to prove Conjecture 1 . Another possible improvement, which would also generalize Theorem 2, is the following.

Conjecture 2. Let $G$ be a connected loopless graph such that $\operatorname{red}(G)$ is none of the four graphs of Fig. 1. Then $\operatorname{cov}_{\mathcal{O}}(G) \leq 3$.

The first inequality of $\left(^{*}\right)$ can be strict in general. We believe this is not the case for the second one.

Conjecture 3. For every loopless graph $G$, it holds that $\operatorname{cov}_{\mathcal{O}}(G)=\operatorname{cov}_{\mathcal{O}}(\operatorname{red}(G))$.

Acknowledgements. I thank J.-S. Sereni for pointing out to me an example when the first inequality of $(*)$ is strict.

\section{REFERENCES}

[1] J.A. Bondy, U.S.R. Murty, Graph Theory, Graduate Texts in mathematics, Springer, New York 244 (2008).

[2] P. Erdös, A. W. Goodman, L. Pósa, The representation of graphs by set intersections, Canad. J. Math. 18 (1966) 106-112.

[3] M. Kano, G.Y. Katona, Odd subgraphs and matchings, Discrete Math. 250 (2002) 265-272.

[4] B. Lužar, M. Petruševski, R. Škrekovski, Odd edge coloring of graphs, Ars Math. Contemp 9 (2015) 277-287.

[5] T. Matrai, Covering the Edges of a Graph by Three Odd subgraphs, J. Graph Theory 53 (2006) $75-82$.

[6] K. R. Matthews, On the eulericity of a graph, J. Graph Theory 2 (1978) 143-148.

[7] L. Pyber, Covering the edges of a graph by..., Graphs and Numbers, Colloquia Mathematica Societatis János Bolyai 60 (1991) 583-610.

[8] A. Schrijver, Combinatorial optimization. Polyhedra and efficiency. Vol. A, Algorithms and Combinatorics, Springer-Verlag, Berlin (2003).

[9] D. B. West, Introduction to graph theory, Pearson Inc (2001).

Department of Mathematics and Informatics, Faculty of Mechanical Engineering,

Ss. Cyril and Methodius University, Skopje,

Karpoš II bB, 1000 Skopje, Republic of Macedonia

Email address: mirko.petrushevski@gmail.com 